\title{
SELF-FORMED, NATURALLY-OPTIMIZED MICROFLUIDIC CHANNELS IN POLYDIMETHYLSILOXANE (PDMS)
}

\author{
Abigail Vinson and Joseph A. Potkay"
}

Advanced Platform Technology Center, Department of Veterans Affairs Medical Center, Cleveland, Ohio, USA

Case Western Reserve University, Cleveland, Ohio, USA

\begin{abstract}
This paper presents initial results for a method to naturally and automatically form microfluidic channels and interconnects through the displacement of a viscous fluid by a gas stream. This new procedure provides an easy means to construct microfluidic networks that automatically and efficiently connect microfluidic channels with low pressure drop and minimal stasis. It is a direct supplement to current microfabrication methods and is in contrast to procedures that utilize complicated construction techniques and complex channel designs. This work demonstrates the concept using PMDS. Fully-formed microfluidic channels created using this approach exhibit round cross-sections, smooth transitions between channels, and achieve the same fluidic resistance as their unmodified counterparts while utilizing $46 \%$ less total channel volume.
\end{abstract}

\section{INTRODUCTION}

Efficient microfluidic channels and routing are critical, and yet remain a challenge, for many lab-on-a-chip systems. In these systems, microfabrication is employed to create microfluidic systems that harness advantageous properties at the micro-scale, such as increased surface-to-volume ratio, reduced sample size, increased transport, or increased surface interactions. However, these systems are often implemented with traditional microfabrication techniques resulting in rectangular cross sections, sharp corners, and abrupt transitions between channels of different dimensions. These properties can be a source of unintended problems. In separation systems such as chromatography columns [1], for example, square cross sections provide dead volume causing peak widening and decreased resolution. Round cross sections are generally preferable. In artificial organ systems [2], non-uniform shear stresses and flow stasis instigate blood coagulation and initiate device failure. For many applications, it is thus advantageous to have channels with round cross sections, rounded corners, smooth transitions between channels of different dimensions, uniform flow distribution and shear stress, minimal stasis, and no turbulence. However, these properties have traditionally been difficult to achieve due to the limitations of lithography-based microfabrication techniques. A method to simply overcome these drawbacks would be highly advantageous, improving microfluidic systems and simplifying the design process.

Routing of fluids to the microfluidic channels is another serious concern. Inefficient fluid distribution networks can use excessive area, create regions of stasis/stagnation, and exhibit flow disturbances, increasing pressure drops. For systems with a large number of microchannels, a branching network is required which efficiently routes fluid from large input and output channels to microchannels where sensing, analysis or modification takes place. In order to address this issue, researchers have attempted to design efficient channel networks or endeavored to mimic biological principles [3] or natural scaling rules, such as Murray's Law [4]. In this paper, instead of attempting to design microfluidic interconnects within the limits of current construction methods, we instead allow nature to automatically optimize their structure.

It has been shown previously [5-7] that when a viscous liquid filling a channel is displaced by a stream of gas, a portion of the liquid remains deposited on the channel walls due to its viscosity. This method can also convert rectangular PDMS cross sections into round ones [7]. In this paper, we extend this technique to produce automatically-optimized, self-formed microfluidic channels and interconnects.

\section{THEORY}

In 1926, Murray investigated the branching of arteries in the human vascular system. He discovered that, in order to minimize biological work, its vessels are connected so that the total flow in the system is carried by a set of vessels whose radii cubed sum to a constant value [4].

$$
\Sigma \mathrm{r}^{3}=\text { constant }
$$

In other words, at each stage of branching, the cube of the radius of a parent vessel equals the sum of the cubes of the daughter vessels [4]. This relationship has additionally been found to apply to other natural branching systems $[8,9]$.

More recently, Murray's law has been generalized to apply to fluidic branching systems with non-Newtonian fluids [10] or those that have other optimality principles [11]. This generalized version of Murray's law replaces the exponent with a constant, C, that is dependent on the properties of the specific system.

$$
\Sigma r^{c}=\text { constant }
$$

In branching systems, it has been generally found that the value of the constant $\mathrm{c}$ has been naturally selected to minimize required work.

\section{DESIGN}

The structure exploited to test this concept is shown in Fig. 1 and is modeled after a generic microfluidic system. That is, in a typical microfluidic system, the fluid is first routed into a large channel with low pressure drop that is large enough to interface

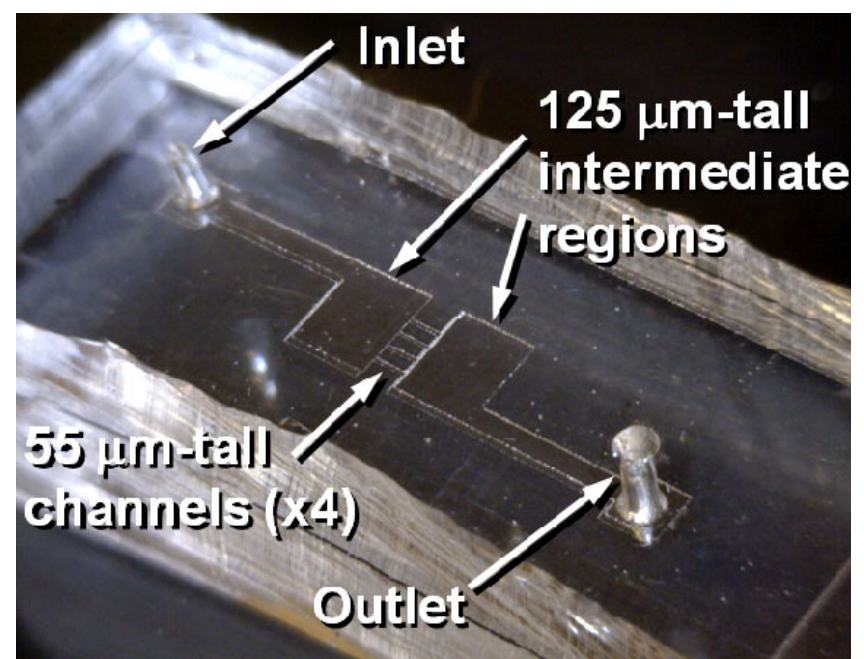

Figure 1: Completed microfluidic test structure. This device is referred to as the "unmodified structure" throughout this paper. 
with the macroscopic world. This large channel then distributes the fluid to anywhere from one to many smaller microfluidic channels that take advantage of properties at the microscale to achieve sensing, analysis, treatment, or separation of components in the fluid, depending on the application. After analysis or modification in the microchannels, the fluid is then routed to a larger channel (with low pressure drop) and can then exit the structure via the device outlet.

This test structure in Fig. 1 was designed to mimic a generic microfluidic system as described above and consists of two 125 $\mu \mathrm{m}$-tall channels connected via four $55 \mu \mathrm{m}$-high center channels. The input and output are separated from the $55 \mu \mathrm{m}$-high center channels through a loosely-bound intermediate region where the auto-optimization occurs. Finally, this test structure was utilized because it exhibits all of the problems associated with standard microfluidic systems constructed using conventional lithographybased techniques: sharp corners, regions of low and high flow, abrupt changes in channel dimension and rectangular cross sections.

\section{FABRICATION}

Microfabrication of the device followed standard PDMS micromolding techniques [12] and proceeded as follows. Masks were created using an image editing program and printed onto a transparency using an Epson Stylus C88+ Inkjet Printer. Microchem SU-8 (2000 series) was utilized to create a mold. Two lithography steps were performed on a 4" silicon wafer to produce both 55 and $125 \mu \mathrm{m}$-tall features on the same mold. A Kinsten KVB-30D UV exposure system was used to cross-link the SU-8. After development in SU-8 Developer, the mold was employed to create PDMS devices. Dow Corning Sylgard 184 was mixed, degassed and poured onto the photoresist mold in a petri dish and cured. After curing, inlet and outlet holes were punched into each device using a $2 \mathrm{~mm}$ diameter needle. The molded PDMS was then bonded to a flat PDMS substrate using oxygen plasma bonding [13] forming an enclosed device. For fluidic access, custom connectors were bonded over the inlet and outlet of the device using a combination of oxygen plasma bonding and PDMS epoxy. A completed device, prior to the attachment of connectors, is shown in Fig. 1.

\section{EXPERIMENTAL}

To begin, the fluidic channels in the structure were completely filled with PDMS using a $10 \mathrm{ml}$ syringe and $5 \mu \mathrm{m}$ syringe filter. The filter removes all large debris and particles which can clog the microchannels during the experiment. Silicone oil (Gelest DMS-T01) can be used to dilute the PDMS and decrease its viscosity [7]. In this work, a 3:1 ratio of PDMS to silicone oil has been employed. A stream of nitrogen gas was then forced through the device at a fixed flow rate using an Omega FMA5508 mass flow controller. At the micro-scale, where gravity and inertial effects are negligible compared to viscous and surface effects, a stable cylindrical gas-liquid interface is formed which minimizes the amount of work required to displace the viscous PDMS inside the device. After steady state was achieved and the gas/liquid interface was stable, channel formation was visually recorded using a BigC Dino-Lite Digital Microscope Camera. The flow rate was then either ramped to investigate higher flow rates or the device was heated to cure the PDMS and fix the microfluidic network. Pressure across the device was monitored using a Honeywell 26PC series pressure sensor and Keithley 2000 digital multimeter. Pressure and flow were converted to fluidic resistance for easier comparison of channel properties at various flow rates.

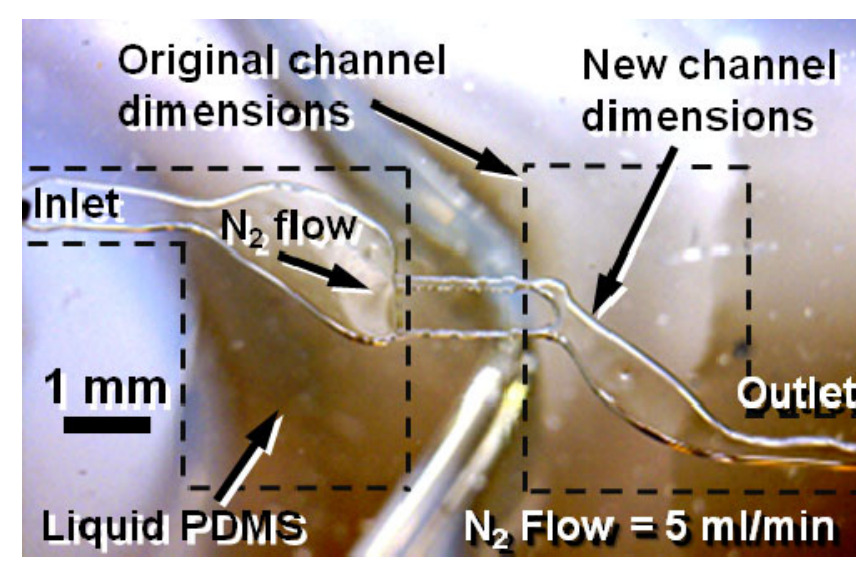

(a)

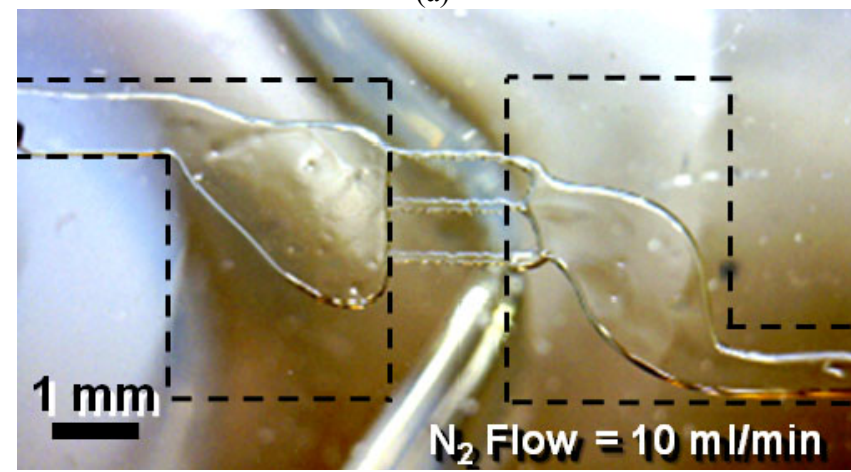

(b)

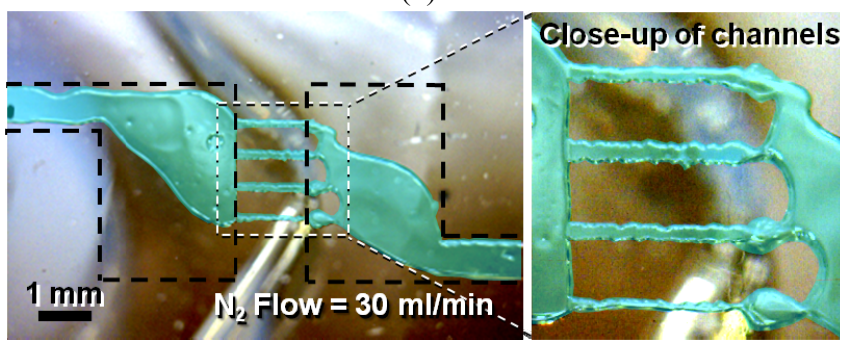

(c)

Figure 2: The structure of the self-formed channels as a function of flow rate. Each of the displayed images has four $55 \mu$-tall channels in the center of the image. However, two of the $55 \mu \mathrm{m}$ channels are not cleared of PDMS until higher flow rates, presumably due to their thinner width (see close-up). In (c), the channel was colored for improved visibility. The unmodified, original channel shape is displayed in Fig. 1.

\section{RESULTS \& DISCUSSION}

Figure 2 shows channel structures formed using this method at three nitrogen flow rates. As can been seen, the process significantly modifies the overall channel structure when comparing unmodified (dotted lines in Fig. 2) to treated channels. In addition, flow channel shapes are interesting and vary considerably from those designed by human researchers. The width of the $125 \mu \mathrm{m}$ inlet and outlet regions and the number of 55 $\mu \mathrm{m}$ channels cleared of PDMS was a function of the $\mathrm{N}_{2}$ flow rate as well as the time exposed to flowing $\mathrm{N}_{2}$ gas. In general, higher flow rates and longer $\mathrm{N}_{2}$ exposure times resulted in larger channel widths and a larger number of clear $55 \mu \mathrm{m}$ channels. All angles within the channel are reduced. Total channel volume for a $\mathrm{N}_{2}$ flow rate of $30 \mathrm{ml} / \mathrm{min}$ decreased by $46 \%$. The $125 \mu \mathrm{m}$-tall inlet region was generally wider than the outlet region suggesting that pressure 

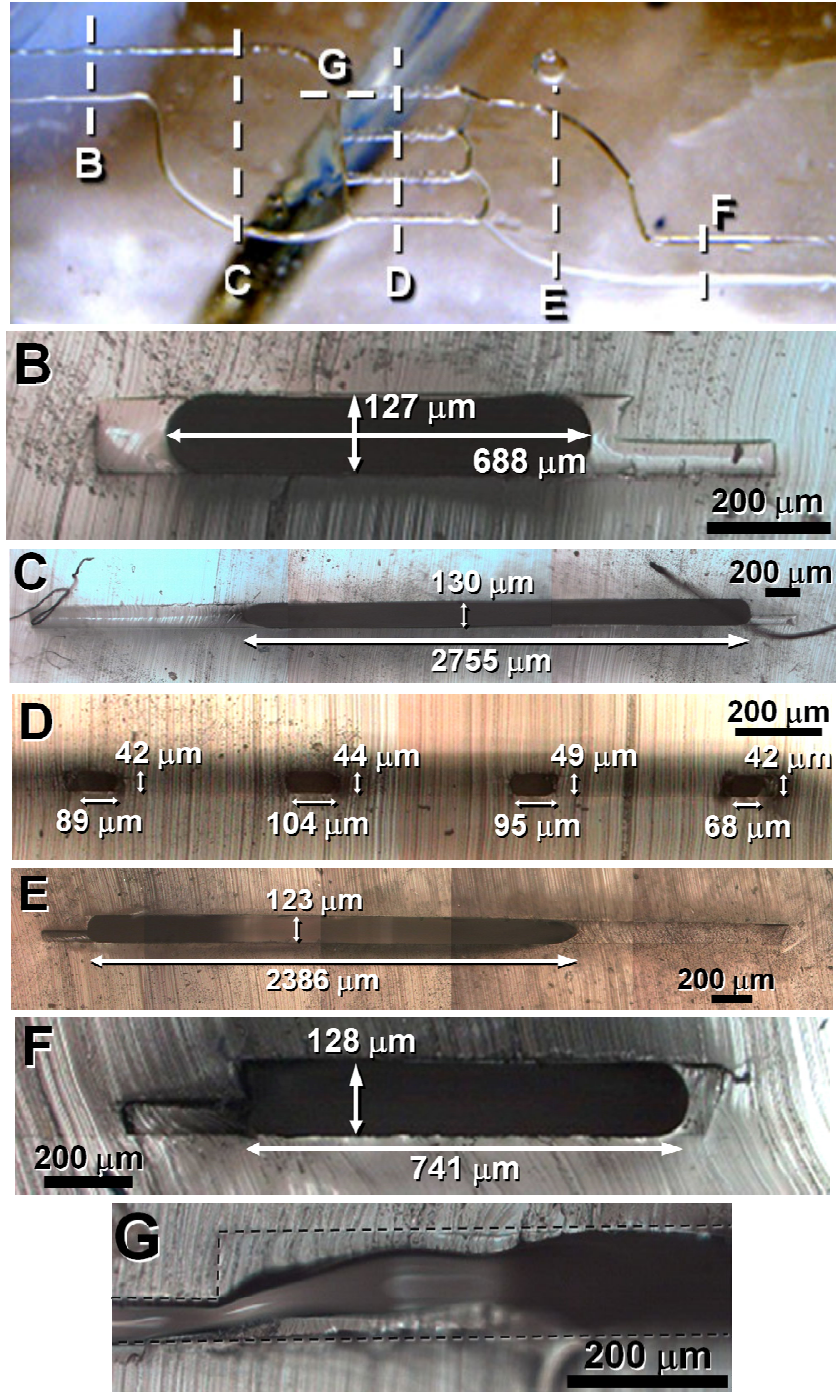

Figure 3: Cross sections of self-formed channels. Letters in bottom images correspond to locations annotated in top image. $G$ shows the transition between the 55 and $125 \mu$ m-high channels. Areas of low flow in the original channel are filled in by PDMS.

has an impact on channel formation. The roughness and differing size of the $55 \mu \mathrm{m}$ channels shows the effect of utilizing inkjet printed masks.

Figure 3 shows cross-sections of the device at various points along the newly-formed channel structure for an $\mathrm{N}_{2}$ flow rate of 30 $\mathrm{ml} / \mathrm{min}$. The process converted all channels from rectangular to round or oval cross sections. Fig. 3a displays another advantage of this method. The smaller rectangular portion of the flow channel on the bottom right is due to mask misalignment and is inefficient for fluid flow due to its smaller height. After processing, this area is completely filled in, eliminating its impact on device performance. Figure $3 \mathrm{f}$ displays a cross section along where the channel transitions from 125 to $55 \mu \mathrm{m}$. Prior to processing, the transition is abrupt and displayed as a dotted line in the figure. After processing, the transition is smooth, resulting in less flow stagnation in the final device.

Figure 4 displays the pressure (Fig. 4a) and fluidic resistance (Fig. 4b) measured across a device during channel formation. Pressure initially increases until nitrogen gas enters the channel structure. It then decreases rapidly and stabilizes as the self-formed

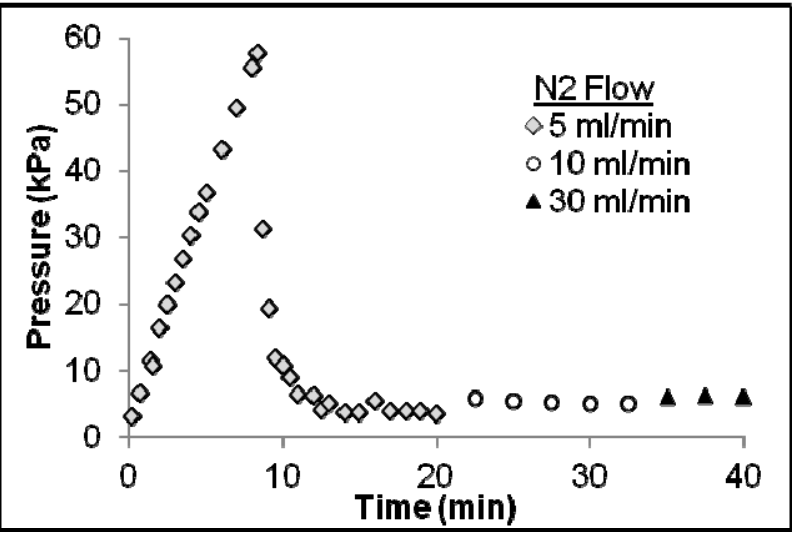

(a)

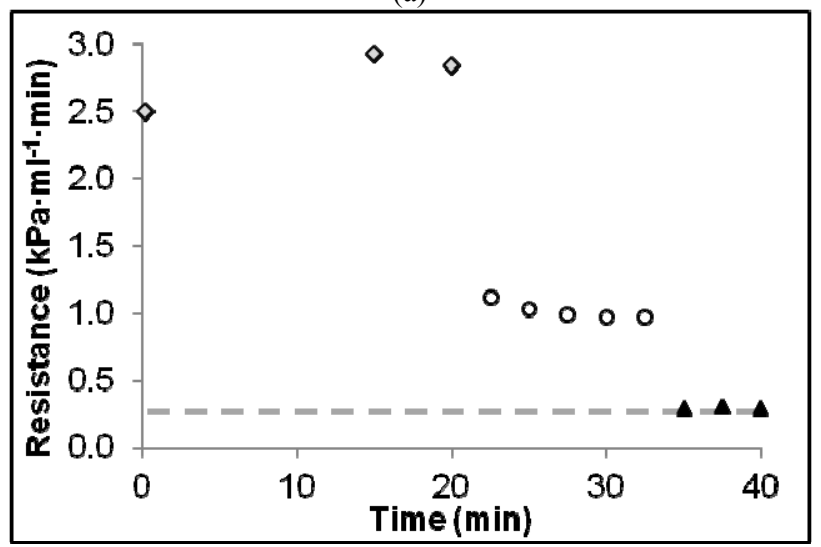

(b)

Figure 4: Pressure (a) and fluidic resistance (b) for a device during the channel formation process. Initial $N_{2}$ flow was set to 5 $\mathrm{ml} / \mathrm{min}$ (open diamonds) until steady state was achieved. It was then raised to $10 \mathrm{ml} / \mathrm{min}$ (open circles) and allowed to settle and then $30 \mathrm{ml} / \mathrm{min}$ (filled triangles). In (b), the y-axis is zoomed-in to show smaller values of fluidic resistance in more detail. At an $\mathrm{N}_{2}$ flow rate of $30 \mathrm{ml} / \mathrm{min}$, the fluidic resistance of the naturally formed channels (filled triangles) is equivalent to that of the unmodified structure (dotted line). The unmodified structure is shown in Fig. 1.

channel structure for that flow rate becomes established. For an $\mathrm{N}_{2}$ flow rate of $30 \mathrm{ml} / \mathrm{min}$, the self-formed fluidic network has the same final fluidic resistance as the initial, uncoated structure. The initial resistance is shown as a dotted line in Fig. 4b.

Table 1 compares channel properties at various points along the microfluidic network as labeled in Fig. 3 and attempts to determine the relationship between flow channels at each level within self-formed channel network. According to Eqn. 2 which describes branching systems that minimize work, the sum of all radii raised to the power $\mathrm{c}$ at each level should be constant. Table 1 thus compares the sum of the hydraulic radii, hydraulic radii squared, hydraulic radii cubed and a term proportional to fluidic conductance to determine the constant c. Since the formed channels are oval in shape and a radius is thus not defined, the channels' hydraulic radii were used in calculations. Only the sum of the hydraulic radii is roughly constant, indicating that the optimality principles for this method differ from those in the natural vasculature $(\mathrm{c}=3)$ [4]. The exact nature of channel structure optimization is still under investigation. 
Table 1: Comparison of self-formed channel properties at various locations annotated in Fig. 3. $\Sigma R_{H}^{3}$ is the sum of all hydraulic radii cubed for all channels at each location in Fig. 3. $W$ and $H$ are the channel width and height. $W H^{3}$ is proportional to hydraulic conductance.

\begin{tabular}{|l|c|c|c|c|c|}
\hline Location & $\boldsymbol{B}$ & $\boldsymbol{C}$ & $\boldsymbol{D}$ & $\boldsymbol{E}$ & $\boldsymbol{F}$ \\
\hline \hline$\Sigma \mathbf{R}_{\mathbf{H}}{ }^{\mathbf{3}}$ & $1.0 \times 10^{6}$ & $1.9 \times 10^{6}$ & $1.0 \times 10^{5}$ & $1.6 \times 10^{6}$ & $1.6 \times 10^{6}$ \\
\hline$\Sigma \mathbf{R}_{\mathbf{H}}{ }^{\mathbf{2}}$ & $1.0 \times 10^{4}$ & $1.5 \times 10^{4}$ & $3.5 \times 10^{3}$ & $1.4 \times 10^{4}$ & $1.4 \times 10^{4}$ \\
\hline $\boldsymbol{\Sigma} \mathbf{R}_{\mathbf{H}}$ & $\mathbf{1 . 1 \times 1 0 ^ { 2 }}$ & $\mathbf{1 . 2 \times 1 0 ^ { 2 }}$ & $\mathbf{1 . 2 \times 1 0 ^ { 2 }}$ & $\mathbf{1 . 2 \times 1 0 ^ { 2 }}$ & $\mathbf{1 . 2 \times 1 0 ^ { 2 }}$ \\
\hline $\boldsymbol{\Sigma} \mathbf{W H}^{\mathbf{3}}$ & $1.2 \times 10^{9}$ & $5.9 \times 10^{9}$ & $2.2 \times 10^{7}$ & $4.3 \times 10^{9}$ & $1.4 \times 10^{9}$ \\
\hline
\end{tabular}

\section{CONCLUSIONS}

In summary, this new method can be used to form naturally optimized microfluidic networks. Furthermore, it converts rectangular cross sections into round ones, eliminates sharp corners, provides smooth transitions between channels of different dimensions, and eliminates channel dead volume. Channel volume decreased by $46 \%$ (compared to the unmodified structure); pressure drop did not increase significantly (within the accuracy of the sensor). Data did not follow Murray's law for natural branching systems. Instead, the sum of hydraulic radii $\left(\mathrm{R}_{\mathrm{H}}\right)$ for all channels at each level was constant. Overall, the process appears to vastly improve the properties of microfluidic channels and routing formed using standard microfabrication techniques.

\section{ACKNOWLEDGEMENTS}

We thank Dr. Ronald Triolo, Dr. Christian Zorman and Mr. Robert (Chris) Roberts for facilities used to complete this work. We additionally thank Mr. Robert (Chris) Roberts for proofreading. This work was supported by Department of Veterans Affairs Rehabilitation Research and Development (VA RR\&D) Grant F7404-R and VA RR\&D Grant C3819C, The Advanced Platform Technology Research Center of Excellence.

\section{REFERENCES}

[1] J.A. Potkay, G.R. Lambertus, R.D. Sacks, and K D. Wise, "A Low Power Pressure- and Temperature-Programmable Micro Gas Chromatography Column," Journal of Microelectromechanical Systems (JMEMS), 16, 5 (2007), pp. 1071-1079.
[2] J.A. Potkay, M. Magnetta, A. Vinson, and B. Cmolik, "Bioinspired, efficient, artificial lung employing air as the ventilating gas," Lab Chip, 11, 17, (2011), pp. 2901 - 2909.

[3] RW. Barber and D.R. Emerson, "Optimal design of microfluidic networks using biologically inspired principles," Microfluidics and Nanofluidics, 4, 3 (2008), pp. 179-191.

[4] C.D. Murray, "The Physiological Principle of Minimum Work: I. The Vascular System and the Cost of Blood Volume", PNAS, 12, 3 (1925), pp. 207-214.

[5] G.I. Taylor, "Deposition of a viscous fluid on the wall of a tube", Journal of Fluid Mechanics, 10, 2 (1961), pp. 161-165.

[6] A. Polynkin, J.F.T. Pittman, and J. Sienz, "Gas displacing liquids from non-circular tubes: high capillary number flow of a shear-thinning liquid", Chemical Engineering Science, 60, 6 (2005), pp. 1591-1602.

[7] M. Abdelgawad, C. Wu, W. Chien, W.R. Geddie, M.A.S. Jewett, and Y. Sun, "A fast and simple method to fabricate circular microchannels in polydimethylsiloxane (PDMS)", Lab on a Chip, 11, 3 (2005), pp. 545-551.

[8] K.A. McCulloh, J.S. Sperry, and F.R. Adler, "Water transport in plants obeys Murray's law," Nature, 421 (2003), pp. 939942.

[9] T.F. Sherman, "On connecting large vessels to small. The meaning of Murray's law." The Journal of General Physiology, 78, 4 (1981), pp. 431-453.

[10] R. Revellin, F. Rousset, D. Baud, and J. Bonjour, "Extension of Murray's law using a non-Newtonian model of blood flow," Theoretical Biology and Medical Modeling, 6, 7 (2009).

[11] G.S. Kassab, "Scaling laws of vascular trees: of form and function," American Journal of Physiology, 290, 2 (2006).

[12] D.C. Duffy, J.C. McDonald, O.J.A. Schueller and G.M. Whitesides, "Rapid prototyping of microfluidic systems in poly(dimethylsiloxane)", Anal. Chem., 70, 23 (1998), pp. 4974-4984.

[13] S. Bhattacharya, A. Datta, J.M. Berg, and S. Gangopadhyay, "Studies on surface wettability of poly(dimethyl) siloxane (PDMS) and glass under oxygen-plasma treatment and correlation with bond strength," JMEMS, 14, 3 (2005), pp. 590-597.

\section{CONTACT}

*J.A. Potkay, joseph.potkay@case.edu; Fax: +1-216-707-6420; ResearcherID: A-1977-2011 